\title{
Ni qué hablar/ni qué decir, ¿construcciones u operadores escalares?
}

\author{
Catalina Fuentes Rodríguez \\ Catedrática de Lengua Española \\ Universidad de Sevilla \\ Dpto. de Lengua Española, Lingüística \\ y Teoría de la Literatura \\ 41004 Sevilla \\ cfuentes@us.es
}

\section{NI QUÉ HABLAR/NI QUÉ DECIR, ¿CONSTRUCCIONES U OPERA- DORES ESCALARES?}

RESUMEN: El presente trabajo se centra en una construcción sintáctica del español que parece desarrollar funciones discursivas delimitadas: ni qué hablar, ni qué decir (+ segmento $\mathrm{u}$ oración). En su construcción incluye la forma $n i$, conjunción coordinante negativa (RAE, 2009, M. Martí, 2020), o variante del operador escalar argumentativo ni siquiera (J. Portolés, 2007). La acompaña un verbo de habla: hablar-decir. Aparece en el español actual como una estructura libre, integrada en la oración, pero también formando parte de dos expresiones fijadas que comparten construcción: [ $n i$ qué+ verbo enunciativo]. Estas expresiones derivan hacia un contenido procedimental, desarrollan una serie de instrucciones para guiar la descodificación del oyente y avanzan hacia su fijación como operador discursivo. Presentan, además, una construcción semilibre con contenido procedimental, ya sea escalar o reafirmativo. El trabajo es cualitativo (describe sus funciones) y cuantitativo (analiza su presencia en los corpus sincrónicos y diacrónico de la RAE).

PALABRAS CLAVE: construcción, pragmalingüística, operador discursivo, macrosintaxis, $n i$ qué hablar-decir.

SUMARIO: 1 . Introducción. 2. Algunos criterios previos. 3. Ni qué hablar/decir. 4. Resultados cuantitativos. 5. Ni que decir tiene.6. Los origenes de las formas: CORDE. 7. Conclusiones.

\section{NI QUÉ HABLAR/NI QUÉ DECIR, CONSTRUCTIONS OR SCALAR OPERATORS?}

ABSTRACT: The present work focuses on a syntactic construction of Spanish that seems to develop delimited discursive functions: ni qué hablar, ni qué decir (+ segment or sentence). This construction includes the form ni, negative coordinating conjunction (RAE 2009, M. Martí 2020), or variant of the scalar argumentative operator ni siquiera (J.Portolés 2007). It is accompanied by a speech verb: hablar-decir. It appears in current Spanish as a free structure, integrated into the sentence, but also as part of two fixed expressions that share construction: [ $n$ $q u e ́$ + verbo enunciativo]. These expressions drift towards a procedural content, develop a series of instructions to guide the listener's decoding, and seem to move towards their fixation as a discursive operator. They also present a semi-free construction with procedural content, whether scalar or reaffirming. The work is qualitative (describing their functions) and quantitative (analyzing their presence in the synchronic and diachronic corpus of the RAE).

KEY-WORDS: Construction, pragma-linguistics, discursive operator, macrosyntax, ni qué hablar-decir.

SUMMARY: 1 . Introduction. 2 . Some previous criteria. 3. Ni qué hablar/decir. 4. Quantitative results.5. Ni que decir tiene. 6. The origins of forms: CORDE. 7. Conclusions.

\section{NI QUÉ HABLAR/NI QUÉ DECIR, CONSTRUCTIONS OU OPÉRA- TEURS SCALAIRES?}

RÉSUMÉ: Le présent travail se concentre sur une construction syntaxique de l'espagnol qui semble développer des fonctions discursives délimitées : ni qué hablar, ni qué decir (+ segment ou phrase). Cette construction inclut la forme ni, soit conjonction de coordination négative (RAE 2009, M. Martí 2020), soit une variante de l'opérateur scalaire argumentatif ni siquiera (J. Portolés, 2007). Elle est suivie d'un verbe de parole: hablar-decir. Elle apparait dans l'espagnol courant comme une structure libre, intégrée dans la phrase, mais faisant également partie de deux expressions fixes qui partagent la construction: [ni qué+ verbe énonciatif]. Ces expressions dérivent vers un contenu procédural, développent une série d'instructions pour guider le décodage de l'auditeur, et elle semble se diriger déjà vers sa fixation en tant qu'opérateur discursif. Ils présentent également une construction semi-libre avec un contenu procédural, qu'il soit scalaire ou de réaffirmation. Le travail est qualitatif (description de leurs fonctions) et quantitatif (en analysant leur présence dans le corpus synchronique et diachronique de la RAE).

MOTS-CLÉS: construction, pragma-linguistique, opérateur discursif, macrosyntaxe, ni qué hablar-decir.

SOMMAIRE: 1 . Introduction. 2 Quelques critères précédents. 3. Ni qué hablar/decir. 4. Résultats quantitatifs. 5. Ni que decir tiene. 6. Les origines des formes: CORDE. 7. Conclusions. 


\section{Ni qué hablar/ni qué decir, ¿construcciones u operadores escalares?}

Catalina Fuentes Rodríguez

\section{INTRODUCCIÓN}

El presente trabajo ${ }^{1}$ se centra en la descripción de una estructura sintáctica del español que parece desarrollar un comportamiento único y funciones discursivas delimitadas: ni qué hablar, ni qué decir (+ segmento u oración). Está formada por dos segmentos: ni, conjunción coordinante negativa (RAE-ASALE, 2009; M. Martí, 1999, 2020) o variante del operador escalar argumentativo ni siquiera (J. Portolés, 2007), y un verbo de habla: hablar-decir. En el español actual aparece con diferentes grados de fijación: a) como una estructura libre, integrada en la oración, y b) formando parte de dos expresiones fijadas que comparten construcción: [ni qué + verbo enunciativo]. Estas expresiones derivan hacia lo procedimental, desarrollan una serie de instrucciones para guiar la descodificación del oyente y nos hacen plantearnos si han avanzado hacia su fijación como operador discursivo. En este momento, el proceso parece estar en sus inicios, con desigual resultado en el caso de las variantes decir y hablar, no solo por el contenido y el tipo de instrucción que vehiculan, sino también por sus caracteristicas distribucionales.

Como corpus, utilizaremos los de la RAE: los dos sincrónicos, CREA desde 1975 a 2000, y CORPES a partir de 2001 hasta 2019. Las etapas anteriores quedan recogidas en CORDE. Recurrimos a él para conocer el posible inicio de esta tendencia.

El estudio es cuantitativo y cualitativo y pretende arrojar luz no solo sobre las formas que estamos considerando sino también sobre la génesis de los operadores discursivos (C. Fuentes Rodríguez, 2003, 2018[2009]), elementos con contenido procedimental (modal, enunciativo o argumentativo) que surgen a partir de estructuras libres. A veces conviven con ellas, o incluso coexisten en situaciones intermedias: la construcción semifijada con parte libre aún y cierto grado de dependencia sintáctica condicionada por los rasgos semánticos del verbo base (C. Fuentes Rodríguez e.p.). Por último, nos interesa ver cuándo aparecen y con qué funciones las dos formas que consideramos. Ello nos permite avanzar en el conocimiento de la historia de los operadores discursivos en general (C. Fuentes Rodriguez, 2020 (ed.)) y nos ayuda a dirimir las distribuciones propias de la categoría frente a los casos aún semifijados.

Nuestra metodología sigue un enfoque de Lingüística Pragmática (C. Fuentes Rodriguez 2017[2000]), teniendo, pues, en cuenta la influen-

1 Este trabajo se ha realizado en el marco del proyecto de investigación FFI 2017-82898$\mathrm{P}$ ("De construcciones periféricas a operadores discursivos, un estudio macrosintáctico del español actual", financiado por Mineco y fondos FEDER, así como dentro de los trabajos del proyecto MacPER: "Macrosintaxis del discurso persuasivo: construcciones y operadores", proyecto de Excelencia PAIDI (P18-FR-2619). 
cia de los factores contextuales en la realización discursiva. Integramos la descripción microsintáctica de las expresiones (sintaxis, entonación y contenido semántico) con la macrosintáctica (comportamiento en el plano modal, enunciativo, argumentativo e informativo), a la vez que relacionamos con el tipo de discurso y registro en el que aparecen.

Para el estudio de su proceso de fijación, nos basaremos en los análisis seminales de gramaticalización de C. Lehman (1985, 2002), C. Company (2004), E. Traugott $(1995,2010)$; E. Traugott y G. Trousdale (2013). Estos últimos autores incluyen la perspectiva de la Gramática de Construcciones (A. Goldberg, 1995, 2003) para fijar el proceso de construccionalización. También nos referiremos a los criterios ya utilizados en otros trabajos para los "operadores en construcción".

Comenzaremos con una breve introducción sobre las características de los operadores, centrándonos, sobre todo, en los reafirmativos. Abordaremos su contenido evidencial y expondremos el paradigma (punto 2). En 3 describiremos ni qué hablary ni qué decir en los diferentes corpus citados. En 4 expondremos los resultados cuantitativos, en 5 trataremos el caso del operador fijado ni que decir tiene y dedicaremos el apartado 6 a mostrar la presencia de las expresiones en CORDE. De este modo, llegaremos en 7 a exponer algunas conclusiones sobre su comportamiento y sobre la fijación de construcciones en general.

\section{Algunos criterios previos}

Como estamos tratando con una construcción (A. Goldberg, 1995), una expresión formada por varios términos que sustentan un comportamiento único ("stored pairings of form and function", A. Goldberg, 2003, 219), debemos aclarar algunos conceptos metodológicos previos. En la investigación que hemos ido realizando dentro del proyecto "De construcciones periféricas a operadores discursivos: un estudio macrosintáctico del español actual" (FFI 2017-82898-P) hemos considerado como relevante y explicativo para el análisis diferenciar 3 estadios:

a) La construcción libre: la distribución puede variar y el contenido surge de la suma de sus componentes manteniendo su valor designativo. Al mismo tiempo la construcción desempeña una función en la oración.

b) La construcción semilibre, en la que se ha producido un primer estadio de gramaticalización: consta de [parte fijada con contenido procedimental + parte libre] (P. Gras y M. Sansiñena, 2015).

c) El operador discursivo: es un elemento gramaticalizado y lexicalizado, que tiene contenido procedimental en alguno de los planos citados y muestra una libertad constructiva en relación con su alcance. No tiene función con respecto al verbo de la oración. 
Aplicamos el concepto de construcción (A. Goldberg, 1995) al estudio de los marcadores discursivos en la misma linea que utilizan E. Traugott y G. Trousdale (2013) el proceso de construccionalización, también citado por N. Evans (2007) en la insubordinación (cuando los componentes iniciales suelen ser conjunciones subordinantes, ahora empleadas con su oración como enunciados libres, o "main clauses").

Tradicionalmente se distingue la sintaxis libre de una expresión del elemento fijado, la unidad fraseológica o gramaticalizada. Se habla de lexicalización y gramaticalización (C. Lehman, 2002), pero para la sintaxis discursiva o macrosintaxis (A. Berrendonner, 1990, 2002; C. Blanche Benveniste, 2003) es muy relevante disponer de un término para denominar las situaciones intermedias en las que una estructura se fija en parte y adquiere un significado único, procedimental.

Por su parte, el concepto de operador que empleamos corresponde más propiamente a los pragmatic markers de B. Fraser $(1996,1999)$. Nuestra propuesta, que llevamos varios años aplicando (C. Fuentes Rodríguez, 2003, 2018[2009], 2016, 2018), difiere de la de B. Fraser en que no consideramos que los pragmatic markers incluyan como un subtipo a los discourse markers. Creemos que hay dos funciones muy delimitadas, con dos comportamientos sintácticos distintos y propiedades distribucionales propias ${ }^{2}$, por lo que extendemos la distinción que J. C. Anscombre y O. Ducrot (1983) aplicaron a los argumentativos, separando conectores (relacionantes entre enunciados $u$ oraciones) de operadores (C. Fuentes Rodríguez, 2003) ${ }^{3}$. Estos se mueven dentro del enunciado pero no dependen del verbo de la oración sino que marcan la referencia a hablante y oyente en sus diferentes planos de actuación (enunciativo, modal, informativo y argumentativo, C. Fuentes Rodriguez, 2013). Seguimos, así, el enfoque modular aplicado en las obras anteriores estableciendo esta diferencia porque, en nuestra opinión, resulta muy rentable desde el punto de vista categorial y macrosintáctico (C. Fuentes Rodríguez, 2019) separar estos dos grupos. Las razones son varias:

- Componen paradigmas diferentes, con unidades y funciones delimitadas.

- Sus funciones discursivas y distribución varian: los conectores enlazan dos segmentos, generalmente dos enunciados o dos oraciones, mientras los operadores tienen su ámbito de acción dentro del enunciado. Su alcance es un segmento de la oración, o bien toda ella. Establecen una indicación sobre su potencial persuasivo, escalar, su focalización informativa o el contenido enunciativo o modal del mismo.

- Sus contenidos procedimentales también son distintos.

\footnotetext{
2 J. Moechsler y A. Réboul (1994:179) afirman: "un opérateur est un foncteur qui a pour argument une proposition atomique, alors qu'un connecteur est un foncteur qui a pour argument une paire ordonnée de propositions".

3 Posteriormente M.Tordesillas (1993) insiste en esta separación.
} 
Tras el estudio de estas dos clases de palabras en el habla coloquial, añadimos otro concepto macrosintáctico relevante: las construcciones semifijadas o híbridas. Los elementos que vamos a estudiar se mueven entre estas dos zonas y nuestro objetivo en este trabajo es delimitarlos. Las conclusiones que obtengamos serán de aplicación a otros elementos de la sintaxis del español en la misma situación.

Para poder hablar de fijación de las construcciones, estas estructuras deben tener movilidad dentro del enunciado, tener un significado único, pues, y este de contenido instruccional.

\section{NI QUÉ HABLAR, NI QUÉ DECIR}

\subsection{Estructura FORMAL}

Estas dos expresiones coinciden en su formación y varian en el verbo que construye su núcleo, aunque ambos son verbos de habla ${ }^{4}$. La construcción investigada es, pues, [ni qué hablar, ni qué decir + segmento u oración]. Pertenecen a la dimensión enunciativa del discurso, a la actividad del sujeto hablante como enunciador, es decir, como responsable del acto de aserción (O. Ducrot, 1984, F. Récanati, 1979, C. Fuentes Rodríguez, 2004).

Incluyen un elemento negativo, ni (RAE, 2009), y una estructura interrogativa indirecta introducida por la forma qué ${ }^{5}$. Coincide, por ello, con otras construcciones que expresan un rechazo: [qué $X$ ni qué $Y . .$.$] o [Ni X ni (+ término malsonante)] (C. Fuentes Rodríguez, e.p.).$ Cabe plantearse, pues, si no podríamos hablar e familia o red de construcciones (Goldberg y Jackendoff, 2004) aunque en este caso forma parte de una estructura mayor (C. Fuentes Rodríguez 2021, e.p.). Ni qué hablar/decir no marcan el rechazo, sino todo lo contrario: la inclusión de un elemento indiscutible, algo que se da por descontado, algo evidente. La construcción sufre un cambio de polaridad y sitúa su alcance en un punto elevado de la escala.

Nuestras preguntas de investigación son varias:

- ¿Cuál es la distribución de ni qué hablar/decir? ¿En qué contextos aparecen? ¿necesitan siempre algo previo?

- ¿Cuál es su contenido procedimental? ¿Es el mismo para ambas formas o presentan variación?

- ¿Está gramaticalizada la construcción? ¿Podemos hablar de operadores discursivos?

Por otra parte, tendremos que precisar el contenido que aportan, que, por lo anunciado, se mueve en el plano de la evidencialidad (V. Plungian, 2010; B. Cornillie y D. Izquierdo (eds.), 2018) y la marcación escalar. El primer concepto es controvertido. Generalmente hace

$4 \mathrm{El}$ estudio de las expresiones metalingüísticas ha ocupado a varios autores. Cabe resaltar la obra de M. Fernández Bernárdez (2002), sobre las combinaciones con decir, así como la publicada por M. Casado, O. Loureda y R. González (2005).

5 A veces aparece en los corpus sin tilde. 
alusión a la indicación del origen de la información obtenida por el hablante. Puede proceder de la propia experiencia, de lo presente en el campo visual, obtenido de oídas, o de manera indirecta a través de otros (discurso referido). El efecto puede ser un distanciamiento de la responsabilidad del hablante (aparentemente, al parecer) o una implicación personal y refuerzo (claro), refiriéndose a que es compartido por toda la comunidad ${ }^{6}$. Estos últimos paradigmas de evidenciales son reafirmativos (por supuesto, desde luego, evidentemente, claro), a la vez que indican modalidad epistémica (J. Nuyts, 2001; L. Ferrari, 2009 \& B. Cornillie, 2009). Esta conjunción hace que sean muy utilizados por el efecto argumentativo que provocan. Actúan como operadores argumentativos escalares en posición elevada. En este campo podríamos situar esta construcción con sus dos formas verbales.

Lo escalar fue invocado hace años por O. Ducrot (1980) al hablar de fuerza argumentativa y se ha desarrollado siguiendo propuestas como las de G. Fauconnier (1975), dirigida al contenido semántico de las unidades (cfr. pp. 361-362). Se rompe, así, la consideración discreta del signo lingüístico de Saussure admitiendo la gradualidad, pero esta no depende exclusivamente de la realidad nombrada, sino de la visión del hablante, de cómo la presenta. Se concreta de manera operativa en un paradigma de unidades, ya convencionalizado, que son los operadores argumentativos:

Un morphème $\mathrm{X}$ est un opérateur argumentatif s'il $\mathrm{y}$ a au moins une phrase $\mathrm{P}$ telle que l'introduction de $\mathrm{X}$ dans $\mathrm{P}$ produit une phrase $\mathrm{P}^{\prime}$, dont le potentiel d'utilisation argumentative est différent de celui de $\mathrm{P}$, cette différence ne pouvant pas se déduire de la différence entre la valeur informative des énoncés de $\mathrm{P}$ et de $\mathrm{P}^{\prime}$ (O. Ducrot, 1983: 10).

En español hay muchos estudios parciales sobre algunas de las unidades que los componen, pero generalmente desde el punto de vista de la coloquialidad. Hace falta una obra inclusiva de todos estos elementos escalares ${ }^{7}$.

\subsection{Funciones}

Estas formas aparecen como enunciados independientes, tras otro anterior, y continúan una enumeración o secuencia de hechos (valor de ni). El introducido por ni qué hablar/decir es el que comparte serie con los anteriores, pero se lo presenta como un elemento superior. Se añade, pues, algo que se muestra como reconocido por todos en su función escalar elevada. Por ello se focaliza, se carga informativamen-

6 Las posiciones en torno a este tema son diversas. Algunos autores separan epistemicidad y evidencialidad y otros unen ambos contenidos. En nuestra opinión son conceptos diferentes: la primera apunta a la modalidad, mientras los evidenciales pueden coincidir con ella o apuntar a la fuente de la argumentación. En este último sentido es en el que aludimos a ese contenido, ya que invocar a la comunidad como fuente de la argumentación y de la información es indicado por estos elementos que estudiamos. Hacemos notar que para otros autores estos elementos no se incluirian en este grupo. Añadimos, sin embargo, que no serian modales reafirmativos en puridad, sino operadores enunciativos que aportan, además, este contenido de refuerzo argumentativo.

7 En preparación. 
te, y, además, adopta un contenido evidencial, en el sentido señalado: no se cuestiona su relevancia y, como consecuencia, se refuerza argumentativamente. Debemos describir en qué se diferencian de otros reafirmativos como por supuesto, desde luego y qué aportan al paradigma. Conviven con su uso como construcción libre, como vamos a exponer a continuación.

\subsubsection{LA CONSTRUCCIÓN LIBRE}

En este primer empleo, activo en la lengua actual, ni qué hablar/decir puede aparecer como una coordinación de negaciones: ni qué hacer ni qué hablar..., dos verbos frecuentes que se repiten.

(1) La ansiedad estaba al máximo y los 66 mil del estadio y los millones en las casas, negocios, estaciones de servicio, fuentes de soda y bares, ya no sabían qué hacer ni qué hablar. (CORPES, I. Pérez Tuesta: Hombres de blanco, 2006).

También lo encontramos en combinatoria no con qué tónico sino formando parte de otra construcción, dependiente de nada:

(2) ¿Se está destruyendo la naturaleza? En Torga florece. "Cuando la sierra y el mar se juntan, no hay nada que hacer ni que decir". (CREA, El Mundo, 19/02/1994).

O intercala una interjección malsonante:

(3) La sacaremos entre yo y Ramón, si quieres, le dijo el Güero, que no hallaba ni qué chingados decir, oigan, al ver a la mujer aquélla achinquechada, haciéndole asi y así a la estufa con las manos como pa ver si tenilla calentura y una chilladera la pobrecita. (CORPES, S.Galindo: El último vaquero, 2005).

Puede introducir un elemento negado en posición escalar inferior, con el uso de ni como variante de ni siquiera, estableciendo el máximo de negación y el mínimo de aserción.

(4) Como respuesta a las denuncias del embajador Alexander, Abdalá, como el gran manejador de los hilos, puede destituir a Manuel Zhindón de Emetel o a Carlos Hidalgo de las Aduanas — de la Lic. Sandra Correa no hay ni que hablar - al tiempo que manda al contralor o al procurador a responderle al diplomático. (CREA, Vistazo, 06/02/1997).

En estos casos ni qué decir / hablar está integrado en la estructura oracional: introducido por una negación o como complemento precedido de un operador argumentativo ni (siquiera). En este último caso ya hay escalaridad. Ni, operador escalar (C. Fuentes Rodríguez, 2018[2009], s.v.), incluye toda la construcción en un punto inferior de la escala negado.

En la construcción libre la estructura es: 
$\mathrm{Ni}$ (conjunción coor dinante u operador escalar)

Verbo (núclęo del predicado)

De que - complemento de ese verbo.

Los verbos hablar y decir mantienen su contenido léxico y su recsión sintáctica.

\subsubsection{LA CONSTRUCCIÓN SEMILIBRE CON VALOR PROCEDIMENTAL NI QUÉ HABLAR/ DECIR}

En este contexto, la construcción adopta un contenido único y se fija como tal estructura, o unidad fraseológica (G. Corpas, 1996) y no permite la inserción de ningún segmento. Además, no funciona ya como complemento oracional. Veamos su distribución.

Ni qué hablar/decir aparece como una expresión única con valor reafirmativo y evidencial. Indica que el segmento que sigue o antecede, su alcance, se sitúa argumentativamente en posición elevada en la escala de lo predicado y es evidente, conocido y aceptado, o discutido. Establece una escalaridad y presenta el elemento situado en posición superior. Se produce, pues, un desplazamiento polar.

Su contenido ya no surge de la suma de sus partes, aunque evidentemente conserva cierta iconocidad, que lo hace identificable, aparte de que sigue funcionando en español como la forma libre. Esto es aceptado por D. Wilson (2016), para quien un contenido procedimental puede convivir con el designativo del que surge, en mayor o menor grado.

También es reconocido por los autores que estudian la gramaticalización (P. Hopper y E. Traugott, 2003; E. Traugott, 2010) para quienes la convivencia de estadios es frecuente. En estas estructuras en proceso es aún más esperable porque son relativamente nuevas en su creación.

Las construcciones en las que aparece esta forma muestra diferentes grados de fijación como paso previo en el proceso que conduce a la gramaticalización de operadores discursivos. En primer lugar, los corpus académicos lo recogen con las dos variantes: qué / que: Ni qué hablar-decir / ni que hablar-decir. Por la pronunciación parece que la forma más adecuada es la primera ${ }^{8}$, pero incluiremos ambas en el análisis, debido a esta vacilación. Los contextos en que aparecen son los siguientes:

- seguido de un complemento: $d e+s n / q u e+o r a c$

- con el alcance antepuesto

- como operador discursivo

El grado de avance de la construcción es distinto en el caso de ni qué hablar y ni qué decir, sobre todo en el avance hacia la función de operador.

En la estructura [Ni qué hablar (decir) de+s.n. /que+oración] hay un elemento escalar (ni qué hablar/decir) que desarrolla contenido procedimental, genera una construcción fijada sin permitir análisis interno.

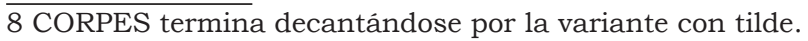


Su función es introducir una información que se añade a una serie anterior. La presentada ahora se considera una aserción más fuerte y menos indudable, más evidente. Ese alcance puede ser una acción, introducida por de que. De marca el régimen del verbo hablar o decir, y que nominaliza la acción para poder complementar al verbo.

(5) A un loco de ésos sólo se lo para con un 38 amartillado sobre su cabeza", había reflexionado Ruibal y era lo único cierto que podía admitir ahora, cuando recorría las arboladas calles de la Villa Colón en busca de un rastro de Mario César Polanski. Ni qué hablar de que Clara ignoraba esta nueva movida de la partida, porque ahora la cuestión la iba a manejar solo y a mi aire. (CORPES, H.Burel: El corredor nocturno, 2005).

(6)Un par de pingüinos en la puerta, con corbatín blanco y todo, solemnes como embajadores inclinaron la cabeza y como si esto fuera poco entraron directamente al comedor, (...), y maniobraron sobre la mesa, pusieron dos servicios, un par de velas, como ocho o nueve cubiertos a lado y lado, vasos, fuentes, un vinito del Rhin que es todo un placer, champagne todo el que quieran, y ni qué decir que las manos del asustado Bagre - ¿Quién va a pagar este menú? - se le iban a las mejillas mientras los ojos estaban a punto de saltar de sus órbitas, se han equivocado de fiesta, caballeros (CREA, R. H. Moreno-Durán, El toque de Diana, 1981).

Aquí la escala se establece entre inferencias. Cuando la expresión ni qué hablar/decir va acompañada de un sintagma es más fácil establecer la organización escalar. En el siguiente encontramos una serie de pintores y aparece tal hiperónimo (pintor) seguido de los nombres de cada uno de ellos: Mabel Arcondo, Emili Aparici, Humberto Battioni, este último en posición escalar superior. Igualmente, en (8) encontramos una serie de museos:

(7) (...) no es raro que despertara también interés en algunos pintores, como Mabel Arcondo, que en su obra La soledad del marino rindió un homenaje a este barrio de procesiones y embarcadizos. O Emili Aparici, quien dedicó innumerables acuarelas al litoral con sus viejos barcos. Ni qué hablar de Humberto Battioni, quien en su casa de la calle Teniente Miranda formara a más de una generación de pintores paraguayos.(CORPES, J. M. Prieto: La ciudad en que vivimos, 2001).

(8) Después están los museos, todos a mano, como el Museo del Ferrocarril y la casa de Bernardino Caballero, también convertida en un museo, tal vez el mejor organizado de la capital. Ni qué hablar del Museo Etnográfico Dr. Andrés Barbero, en la avenida España y Mómpox. (ídem).

En (9) no aparece ningún hiperónimo, sino diferentes elementos del mismo campo semántico: canto judio- campana de los cristianos- concierto de burros. 
(9) —iQuién puede meditar con tanta alharaca! —rebuznó el Burro impertinente - prefiero mil veces la algarabía del canto judío o las tristes campanas de los cristianos, ni que decir del concierto de mis congéneres. (CREA, J. L. Najenson: Memorias de un erotómano y otros cuentos, 1991).

Ambas formas pueden aparecer también acompañando al último segmento de una enumeración. Introducen el elemento más alto, en contexto positivo o negativo, según lo anterior. A veces van precedidos de una coordinación que marca claramente la adición:

(10) "Prefiero llorar su muerte lejos de las cámaras y micrófonos, y ni qué hablar de los paparazzi con sus agresiones", ratificó el cantante memorando el asedio del que fue víctima al divorciarse de su mujer. (CREA, La Nueva Provincia, 04/09/1997).

Los paparazzis se colocan en una posición superior. Todos comparten la idea de no querer que lo graben en su intimidad. Igual ocurre en (11): se relacionan los avisos discriminatorios con las oportunidades, estas por encima en la escala. El sentido en todos los casos es "no hace falta decirlo, es evidente".

(11) (...) lo paradójico del caso es que en los debates con los medios televisivos, éstos se ampararon en la "libertad" para continuar emitiendo programas que denigran la cultura y vestuario de un vasto sector de la población peruana. Esto continúa hasta el día de hoy, los avisos discriminatorios también, y ni qué decir de las oportunidades. En suma, no se ha avanzado mucho (CORPES, R. Aguilar Vizcarra: "Parte I. Racismo, prejuicio y discriminación, una mirada desde nosotros, 2003).

Esta construcción, como por supuesto o claro, no solo intensifica, sino que, además, presenta lo dicho como algo indiscutible y evidente. $Y$, frente a estos elementos, añade un segmento que se coloca escalarmente en posición superior. Equivale a "no hace falta decir, mencionar". En francés equivale a il va sans dire... (Ramos Sañudo, 2017).

(12) Ninguno de ellos tendría dinero suficiente para sobrevivir sin trabajar más de un par de semanas, ni qué hablar de pensiones o jubilaciones. Ninguno de ellos tendría ahorros suficientes para garantizar la educación de sus hijos. (CORPES, F. Reyes-Heroles: El abismo, 2002).

(13) Él estuvo en menos de dos minutos en tu casa. Ni qué decir que fue el consuelo perfecto. Ni qué decir que hizo todo para calmarte y tú, ingenua y boba. (CORPES, V. Cordero : Voces, 2011).

En ocasiones el enunciado es exclamativo. Este aspecto mirativo (A. Aikhenvald, 2005 y S. De Lancey, 1997), de asombro, se añade a la intensificación que supone la posición escalar.

(14) La luminosidad solar continuará ascendiendo y en algún momento evaporará los océanos y calcinará los continentes. ¡Ni qué hablar del calentamiento global! Nuestro planeta quedará devastado. (CORPES, D. R. Altschuler: Hijos de las Estrellas, 2002). 
Junto a todos estos ejemplos con de, (15) incluye una circunstancia hipotética, que se propone como superior escalarmente, o en (16) "con relación a", un punto de referencia.

(15) Así podriamos recorrer historias recientes de diferentes países a los que muchos están pensando en irse. Ni qué hablar si se piensa en Estados Unidos. (CORPES, "TRIBUNA ABIERTA". Clarín. Buenos Aires: clarin.com, 22-01-2002).

(16) En promedio, el sueldo de los trabajadores registrados es de apenas 230 dólares mensuales -equivale a 1,35 dólar la hora-, por debajo de Brasil y menos de la mitad de México. Y ni qué hablar con relación a los países desarrollados. En Europa el sueldo promedio ronda entre 1.500 y 2.000 dólares, según los países. (CORPES, "Los sueldos argentinos son los más bajos de Latinoamérica». Clarín, 1911-2002).

Todos estos casos incluyen complementos de los verbos hablar y decir. Y, por tanto, muestran que está aún latente el significado originario designativo, así como la estructura sintáctica de dependencia que mantiene en la estructura libre. Pero ¿podremos decir que ha empezado el proceso de fijación o, por el contrario, estamos ante una situación intermedia en la que se ha producido el cambio a contenido procedimental antes que el cambio sintáctico?

En primer lugar, esta construcción utiliza las formas enunciativas decir, hablar, para construir un elemento que desarrolla un contenido procedimental escalar. Este contenido los acerca a los operadores argumentativos. La base léxica, sin embargo, es enunciativa.

Por otro lado, se recurre a una formulación interrogación + negación. El sentido sería: "no habria nada que decir o nada que hablar del segmento que sigue". Y esto nos lleva a su distribución sintáctica y a preguntarnos sobre su función dentro del enunciado. Esta construcción (con sus dos variantes) va seguida de un complemento ligado al verbo decir o hablar por sus características sintácticas: hablar de, decir de. O bien que, regido por el verbo decir. En (6), "y ni qué decir que las manos de...", estamos ante una enumeración, por ello generalmente lleva $y$, pero se trata de una serie de hechos que podrian haberse introducido sin que. Este que viene regido por el verbo. Por tanto, tenemos que pensar que no estamos en estos casos ante un operador ya gramaticalizado, desgajado del resto de la oración, sino ligado a ella. No es el mismo caso que por supuesto, que se combina con que o sin esta conjunción y alternan en el mismo ejemplo. Aquí no podríamos eliminar la forma que. Lo mismo podría decirse cuando va seguido de la preposición de, también regida por este verbo: "ni qué decir del concierto de mis congéneres" en (9). En este caso, sin embargo, podemos encontrarnos una enumeración de sintagmas nominales, sin la preposición. Sería factible y aceptable: "ni qué decir el concierto de mis congéneres". Este caso parece más avanzado hacia el uso como operador.

Hasta ahora, pues, podemos decir que estamos ante una construcción aún ligada sintácticamente al elemento que constituye su alcance, y por ello puede introducirlo con que o de, pero también puede 
combinarse con otros sintagmas libres sin preposición.

Su contenido, sin embargo, es ahora procedimental: escalar, introduce un elemento superior en la escala a los anteriormente enumerados y se presenta como algo indiscutible, se reafirma y se plantea como conocido. Podríamos afirmar para él un contenido evidencial.

Esta situación que hemos ejemplificado es la habitual. De hecho, en una búsqueda en CORPES, los resultados hablan por sí mismos.

Los 53 casos de ni qué hablar se distribuyen del siguiente modo:

A) Manteniendo su valor de estructura libre: 49 .

1. Con extensión: de, con, si: 48

2. Coordinante: 1

B) Operador: 4 (pospuesto: 2. De ellos uno intercalado y otro en inicial).

Predomina claramente el uso integrado: 48 de 53 casos. Y en CREA 13 de 15 casos. En ni qué decir la tendencia es semejante, aunque con más casos en CORPES: 159 casos de un total de 242. En CREA 25 de 66 casos.

\subsubsection{El USO COMO OPERADOR}

Como acabamos de ver, son menos frecuentes los casos de fijación como operador, lo que nos hace pensar en una construcción aún en proceso. En este empleo ni qué hablar y ni qué decir tienen un comportamiento distinto.

3.2.3.1. Los 4 casos de CORPES de ni qué hablar muestran diferentes situaciones. El ejemplo siguiente es un caso de posposición a su alcance. Este no es un complemento literalmente, ha perdido la preposición. Ni qué hablar mantiene valor evidencial y escalar, aparece en posición final, por lo que podría considerarse cercano ya al uso como operador: [alcance + ni qué hablar].

(17) - Dígame Lourdes ¿Cómo fue que viniste a vivir en Buenos Aires? Pues como me parece, tu país es mucho mejor para vivir, además te oigo siempre hablar con tu hija en una lengua que no es el español y las músicas que escucho ni qué hablar (CORPES, J. A. Delgado: Yvate, 2003).

Este elemento aparece pospuesto a un sintagma ("las músicas que escucho"), que constituye un elemento superior en la escala a los otros argumentos: "tu país es mucho mejor para vivir, o la lengua". Igual en esta enumeración de compositores:

(18) Estos tendrian que escuchar un poco más a Bach, a Beethoven ni que hablar. Carancho. Eso eso eso eso eso... (CORPES, M. Garcíal: Zitarrosa intimo, 2014). 
Como podemos ver por estos dos ejemplos, es claro el proceso de anteposición del alcance, que, al desligarse sintácticamente, pierde la preposición. Al mismo tiempo se evidencia que, aunque sean varios elementos organizados en escala, funcionan como dos enunciados distintos: "tendrian que escuchar más a Bach. Y a Beethoven ni qué hablar".

Tenemos otros casos de ni qué hablar en posición inicial, afectando a todo el enunciado, y en posición intercalada. En ellos es más claro el valor intensificativo, y se propicia una interpretación como reafirmación, ya que los elementos no están ordenados escalarmente. En (19) afecta a todo el enunciado:

(19) Dallas viene de tres partidos sin perder. En la más reciente fecha de la MLS, venció 4-1 de visitante al Dynamo de Houston. "Ni qué hablar, es un juego especial, (El Galaxy) es el último campeón y, aunque respetamos a todos, jes Los Ángeles!”. (CORPES, J. García: Al día Dallas. Dallas: aldiadallas.com, 08-05-2015).

En este caso no se explicita la escala, pero parece adquirir un valor reafirmativo o de comentario de fuerza semejante a por supuesto. En la posición intercalada sí refuerza el último miembro de la enumeración: "qué impacto determina en la práctica clínica".

(20) Como corolario final de lo anterior, no tenemos un lugar confiable que nos permita acceder a información adecuada sobre qué investigación clínica se produce, quiénes la hacen, dónde se publica, quién la financia y, ni qué hablar, qué impacto determina en la práctica clínica en nuestro país. (CORPES, A. Danza, Revista Médica del Uruguay. Montevideo: rmu.org.uy, 09-2015.

Hemos hecho una búsqueda en CREA de 1975 hasta 2000, ya que CORPES empieza en 2001 y comparte formas con CREA. Encontramos en este último corpus 11 casos, 6 a inicio de enunciado y 5 intercalados. De ellos, 9 son de la función a) seguida de un complemento con de, una oración con que, o un sintagma. Tenemos dos casos en que aparece intercalado, como un marcador, o pospuesto en otro. En este se muestra su disposición evidencial, reafirmativa y manteniendo lo escalar.

Encontramos un caso en que se emplea como forma independiente, cercana a un operador discursivo, pero no en respuesta:

(21) No habrá un himno porque los croatas católicos tienen el suyo, los bosnios musulmanes cantan el propio y los serbios ortodoxos, ni qué hablar, no están dispuestos a entonar estrofas ajenas.(CREA, Clarín, $11 / 04 / 1997$.

Sin embargo, en el pospuesto la situación es semejante a la de CORPES, lo que se antepone es el tema.

(22) Había mucho que hacer en ese sentido, pues, la ciudad de Buenos Aires desde su fundación, entre otros problemas, había carecido de nivelación, y eran muy pocas las calles en buen estado; las veredas malas, estrechas, construidas la mayor parte de mal ladrillo. De las aceras ¡ni qué hablar! (CREA, F. Arenas: Cómo era Buenos Aires, 1979). 
Aparece en primera posición el complemento que constituye su alcance y se mantiene la preposición régimen de. La tabla siguiente muestra los datos cuantitativos para ni qué hablar en los dos corpus:

\begin{tabular}{|c|c|c|}
\hline Ni qué hablar & CORPES 53 casos & CREA: (1975-2000): 15 casos \\
\hline Coordinante o con $n i$ escalar & $1(1,88 \%)$ & 0 \\
\hline $\begin{array}{l}\text { Construcción semilibre con } d e \text {, } \\
\text { que, si... }\end{array}$ & $48(90,56 \%)$ & $13(86,66 \%)$ \\
\hline Operador: Pospuesto & $4(7,54 \%)$ & $2(13,33 \%)$ \\
\hline
\end{tabular}

Tabla 1: Ni qué hablar en CORPES/CREA

\subsubsection{Ni qué decir, por su parte, es más complejo en su com- portamiento y registra más ocurrencias}

Encontramos más casos en que parece emplearse el valor coordinativo de ni o con el valor escalar de ni siquiera.

\begin{tabular}{|c|c|c|}
\hline Ni qué decir & CORPES: 242 casos & CREA: (1975-2000): 45 casos \\
\hline $\begin{array}{c}\text { Coordinante o con ni esca- } \\
\text { lar+extensión }\end{array}$ & $66(27,27 \%)$ & $18(40 \%)$ \\
\hline Construcción con de, que, si... & $159(65,70 \%)$ & $25(55,55 \%)$ \\
\hline Operador: Pospuesto & $17(7,02 \%)$ & $2(4,44 \%)$ \\
\hline
\end{tabular}

Tabla 2: Ni qué decir en CORPES/CREA

$\mathrm{Ni}$ qué decir muestra 242 casos en CORPES. De ellos la mayoría son del primer uso como construcción libre que mantiene su valor léxico y la construcción semifijada, y pocos como operador, pero con alguna variante con respecto a ni qué hablar. Encontramos muchas más ocurrencias en la coordinación negativa o con el marcador escalar, aunque proporcionalmente baja en el uso como operador.

Ni qué decir aparece en más ocasiones como operador y parece más desarrollado. Lo encontramos acompañando al último término de una enumeración, reforzando su contenido escalar:

(23) Nosotros los hombres, hemos sido tontos porque siempre tratamos de entenderlas y cambiarlas, cuando lo más lindo es amarlas y privilegiado aquél que conquiste su admiración, su respeto, su cariño y ni que decir SU AMOR. (CREA, La Hora, 04/01/1997).

En muchos casos aparece pospuesto al último segmento de la serie, acompañado de la conjunción copulativa $y$. Se citan otros elementos anteriores, a los que se une el introducido por ni qué decir como el situado en posición escalar superior. De este modo se reafirma:

(24) Los alemanes lloraron, y los latinoamericanos ni qué decir. No era para menos. (M.Benedetti: Primavera con una esquina rota, 1982). 
(25) Que semanas antes viajan a Miami, New York y París y se traen lo último de la moda, vestidos cuyo valor triplica el salario anual de cualquier profesional. Y las joyas ni qué decir, les faltan dedos y cuellos para lucirlas. (CORPES, H. Carvalho Oliva: La ciudad de los inmortales, 2005).

En otros contextos, su función discursiva parece acercarse más a la de un evidencial que afecta a toda una aserción, ya sea la contenida en todo el enunciado o en una oración. Ocurre generalmente cuando aparece intercalado, sin complemento, distribución propia de un operador discursivo. Afecta a todo el enunciado. Establece el refuerzo de esa aserción, presentándola como algo indiscutible y evidente:

(26) Esta breve aparición, ni qué decir, rendia sus frutos. En los pocos segundos que la puerta de Noemí permanecía abierta, el público, incluido Luis Alberto, se daba maña para reconocer sus encantos. (CORPES, F. Ampuero: Puta linda, 2006).

En ocasiones, aunque aparece intercalada en el enunciado, en realidad afecta al conjunto oracional que sigue en coordinación e indica posición elevada, equivalente a la reafirmación:

(27) Poco después de su sonado éxito con el caso Altozano le salió un contrato en Nueva York y, ni qué decir, agarró maletas y un peruano más salió al mundo. (CORPES, O. Salomé: La armonía de los mapas. Lima: Zignos, 2007).

Con este mismo valor encontramos la expresión ni qué decirlo, en distribución parentética, intercalada o final, afectando a todo lo dicho en el enunciado o en la oración. Podría pensarse que, al mantener el clítico referido a toda esta aserción, constituiría un estadio previo. Encontramos 4 casos de esta combinatoria:

(28) Tras someterse a largas y penosas sesiones de hipnosis, cientos de personas han recordado, de pronto, los abusos sufridos durante su niñez a manos de sus profesores, tíos, hermanos e incluso de sus padres y madres. En algunas ocasiones, ni qué decirlo, se trataba de auténticas anamnesis: en el clásico esquema freudiano, los sujetos habian cancelado los sucesos debido a su carga traumática. (CORPES, J.Volpi: Leer la mente, 2011).

Su distribución es semejante a la de ni qué decir. En el ejemplo anterior se intercala y afecta a todo el enunciado, pero también admite la posición final y la inicial.

(29) Para no mencionar, reconozcámoslo de una vez su poder terapéutico. Los afectos cierran heridas, ni qué decirlo. O les echan sobre su superficie purulenta un poco de ceniza. (CORPES, M. Valdovinos: Lihn, la muerte, 2012).

(30) Se inventó una biografia propia porque no quería vivir la trazada por su familia. La hizo a golpes de palabras, de poesía, de mundos imaginarios, todo un artista. Ni qué decirlo, afrancesado. (CORPES, idem). 
El contenido transmitido es claramente evidencial y a la vez reafirmativo: está fuera de toda duda, no es necesario mencionarlo, es evidente, todos pueden saberlo o percibirlo. El elemento escalar argumentativo deriva hacia la reafirmación evidencial.

En cuanto a su sintaxis, se acerca a la de un operador, sobre todo cuando lo encontramos constituyendo solo una intervención reactiva, como confirmación de una aserción:

(31) —Jodido ser burro - filosofó al rato Jimmy.

-Ni qué decirlo - asintió Pelota. Terminó su cerveza de un trago

y dijo-: ¿vamos yendo?

(CORPES, Ferrini, Ernesto: La tristeza de los burros. Lima:

Planeta, 2006.

En este contexto aparece con el complemento objeto, pero actúa como unidad independiente, como un enunciado que constituye intervención. Aquí su comportamiento es equivalente al de un disjunto (S.Greenbaum, 1969; R. Quirk et al., 1972), es decir, un adverbio u operador modal (C. Fuentes Rodríguez, 1991, 2018[2009]), aunque mantiene el clítico. Su empleo no está muy extendido, como vemos, frente a la construcción con extensión léxica. Ambos aspectos indican un proceso en marcha, que podría fijarse en un futuro.

\section{Resultados cuantitativos}

Con respecto a los datos cuantitativos, resalta claramente el dominio de la forma ni qué decir sobre ni qué hablar. El contenido más genérico como verbo de habla de decir puede haber ayudado en este sentido. Las proporciones, sin embargo, entre el uso con complemento léxico frente al desarrollo como operador coinciden. Podemos hablar de una construcción semilibre: ni qué hablar / decir + de, que + alcance, con una parte libre y otra fija. Esta desarrolla un contenido procedimental, de evidencia, refuerzo y escalaridad. Introduce un elemento superior en la escala a otros anteriores y muestra que este elemento es indiscutible y evidente frente a los demás. Como efecto, lo reafirma. Este contenido procedimental de refuerzo escalar y evidencia se une en el operador a una distribución sintáctica en la que aparece sin extensión, formando grupo entonativo independiente, con movilidad, anteponiéndose o posponiéndose a su alcance, cuando es un segmento, y en ocasiones intercalado cuando afecta a todo el enunciado.

En ni qué decir, en estos casos, transmite un contenido evidencial, ya no escalar, y se orienta hacia la reafirmación. Semejantes serian: por descontado, por supuesto.

\begin{tabular}{|c|c|c|}
\hline & $\begin{array}{c}\text { CORPES: Construcción con } \\
\text { régimen/operador }\end{array}$ & $\begin{array}{c}\text { CREA: Construcción con régi- } \\
\text { men/operador }\end{array}$ \\
\hline Ni qué hablar & $48 / 4$ & $13 / 2$ \\
\hline Ni qué decir & $159 / 17$ & $25 / 2$ \\
\hline
\end{tabular}

Tabla 3: Comparación de ambas formas en CORPES/CREA 
A estos datos tenemos que añadir los casos en que estas formas no aparecen con tilde. Esta vacilación se percibe en CREA, ya que CORPES unifica a la forma con tilde.

\begin{tabular}{|c|c|c|}
\hline CORPES & Ni qué hablar: 55 & Ni qué decir: 263 \\
\hline Coordinante o con ni escalar & 1 & 75 \\
\hline $\begin{array}{c}\text { Construcción con comple- } \\
\text { mentos }\end{array}$ & 49 & 26 \\
\hline Operador & 5 & 26 \\
\hline
\end{tabular}

Tabla 4: Funciones de las formas en CORPES

\begin{tabular}{|c|c|c|}
\hline CREA & $\begin{array}{c}\text { Ni qué hablar/ni que hablar: } \\
15 / 6\end{array}$ & $\begin{array}{c}\text { Ni qué decir/ni que decir: } \\
33 / 13\end{array}$ \\
\hline Coordinante o con ni escalar & $0 / 2$ & $19 / 3$ \\
\hline $\begin{array}{c}\text { Construción con complemen- } \\
\text { tos }\end{array}$ & $13 / 2$ & $8 / 8$ \\
\hline Operador & $2 / 2$ & $6 / 2$ \\
\hline
\end{tabular}

Tabla 5: Funciones de las formas en CREA

En ni qué hablar/decir es más frecuente la tilde, como podemos comparar en CREA. La unificación de CORPES no se extiende a ni que decir tiene, que es más frecuente sin ella.

\section{NI QUE DECIR TIENE}

No podemos dejar de tratar, en relación a ni qué decir, el desarrollo del operador ni que decir tiene, que puede utilizarse como segmento aislado entonativamente antepuesto o pospuesto a su alcance.

En (32) aparece intercalado, afectando en todo el enunciado y en (33) se pospone a su alcance, ocupando la posición final:

(32) Esa minimización de los méritos propios, que se estilaba antes, ya no se lleva. La humildad, ni que decir tiene, es mentira. Mas si es cortés, sí que se agradece. (CREA, El Mundo, 01/06/1995).

(33) En uno de los números podían salir las dos juntas. Con vestidos nuevos, ni que decir tiene. Vestidos de revista. (CREA, F. Fernán Gómez: El viaje a ninguna parte, 1985).

En este empleo actúa claramente como un operador reafirmativo, evidencial. No aparece el valor escalar, ni necesita una serie previa.

En C. Fuentes Rodríguez (2018[2009] se recoge con el valor de un operador modal: "Marcador de ausencia de duda. Indica enfatización de lo dicho y evidencia (...). Es un reafirmativo coloquial y propio de la lengua oral" (s. v.). En su comportamiento en el plano argumentativo se reconoce su función como marcador de fuerza argumentativa, así como enfatizador del enunciado o segmento afectados. Recoge el valor polifónico ya que aparece como "una marca clara del locutor como enunciador, pero al mismo tiem- 
po señala como evidente y compartido por la comunidad lo dicho" (s.v.).

$\mathrm{Su}$ presencia en los corpus es mayor que la forma ni qué decir. En CORPES la proporción es: ni qué decir 263, todos con tilde, ni que decir tiene 241, frente a 32 con tilde. En CREA los datos muestran la misma proporción: 165/3. Es un operador evidencial que reafirma lo dicho, como fuera de toda duda, tiene movilidad, un contenido procedimental definido, y está fijado como operador. Nos preguntamos, por ello, si ha frenado este elemento el desarrollo de las otras formas consideradas.

\section{LOS ORÍGENES DE LAS FORMAS: CORDE}

El análisis del corpus diacrónico de la RAE nos permite rastrear su uso hasta épocas tempranas.

\subsection{Ni QUÉ hablar APARECE EN UN USO LÉXICO EN EL S. XVII:}

(34) 10. ¡Oh Señor, y quién podrá decir del gusto que un alma recibe cuando se ve fuera de estas troneras, zaquizamies y camaranchones del mundo! No sabe qué decir ni qué hablar en esta ocasión. (CORDE, a 1613, S. Juan Bautista de la Concepción (Juan García López)).

Posteriormente, encontramos algunos casos en el s. XIX y XX:

\begin{tabular}{|c|c|c|c|}
\hline NI QUÉ HABLAR & Construcción libre & Con complementos & Operador \\
\hline $\mathrm{XIX}$ & 0 & 1 & 1 \\
\hline $\mathrm{XX}$ & 1 & 3 & 5 \\
\hline
\end{tabular}

Tabla 6: Ni qué hablar en CORDE según funciones

Los resultados totales, sin separar siglos, por construcción, son:

\begin{tabular}{|c|c|c|}
\hline CORDE & Ni qué hablar & Ni que hablar \\
\hline Construcción libre & 2 & 3 \\
\hline Con complementos & 4 & \\
\hline Operador & 6 & 2 \\
\hline
\end{tabular}

Tabla 7: Comparación de ambas variantes en CORDE

Son pocos casos, pero ya localizamos el uso de la construcción fijada como operador a finales del s. XIX. Del uso con complementos encontramos el siguiente:

(35) Del sol, de la lluvia y del enemigo, ni qué hablar; entre todos los obligaban a no descansar, a olvidarse de lo que es el sueño y a familiarizarse con la muerte. (CORDE, F.Gamboa: Suprema ley, 1896).

Del operador también localizamos un caso en respuesta en el s. XX: 
(36) Rudelinda ¿Contamos con usted, entonces?

Na Martiniana ¡Phsss! Ni qué hablar. (Rudelinda mutis.) (CORDE, F. Sánchez: Barranca abajo, 1905).

Las formas sin tilde aparecen menos en CORDE y corresponden a la construcción: no hay ni que hablar, que estaria en su origen, y que indica claramente el valor de conocido. De 1800 en adelante se registran 3 casos, 2 de esta perífrasis, con ni operador escalar y uno de la construcción funcionando ya como operador.

(37) Allá no es que yo fuese un duque, ya lo sabemos, pero uno era alguien, me parece a mí. Tocante a caceríos, no hay ni que hablar. (CORDE, M. Delibes: Diario de un emigrante, 1958).

(38) Juan Luis: Y me he propuesto que se den un abrazo. Dos buenos criollos * como ustedes no pueden vivir así, enojados. De parte de Butiérrez, ni que hablar...

Don Zoilo (muy irónico): ¡Claro! ¡Ni que hablar! ¡Mande no más, amigazo! Usted es muy dueño. Vaya y digalé a ese buen mozo que se apee... Yo voy a sujetar los perros... (CORDE, F.Sánchez: Barranca abajo, 1905).

\subsection{Ni QUE DECIR ES MÁS FRECUENTEen CORDE, EN AMBAS VARIANTES}

\begin{tabular}{|c|c|c|}
\hline CORDE & Ni qué decir & Ni que decir \\
\hline Construcción libre & 34. Casos desde el XVI & $\begin{array}{c}\text { 3. Son del XIX-XX. Valor } \\
\text { reafirmativo }\end{array}$ \\
\hline Con complementos & 3 & 13 \\
\hline Operador & 16 & \\
\hline
\end{tabular}

Tabla 8: Ni qu(é) decir en CORDE

Ni que decir tiene es el más frecuente en las variantes con tilde ${ }^{9}(7)$ y sin ella (69). Esta última se impone como en la etapa actual.

En resumen, los datos, según tipo de construcción, en CORDE son los siguientes:

\begin{tabular}{|c|c|c|c|c|}
\hline NI QUÉ DECIR & C. libre & Con complem. & Operador & Ni qué decir tiene \\
\hline XVI & 1 & & & \\
\hline XVII & 1 & & & \\
\hline XVIII & 1 & & & \\
\hline XIX & 16 & & 1 & \\
\hline XX & 18 & 1 & 2 & 2 \\
\hline
\end{tabular}

Tabla 9: Ni qué decir en CORDE

No aparece en inicial hasta el s. XIX. El primer uso de operador data de 1851, pero es un único caso. En el XX aparecen 2. Estas

$9 \mathrm{Ni}$ qué decir tiene sí aparece con complementos: “Todas las mañanas a las nueve la muchacha sale para "La Tribuna" con mi artículo con ilustraciones; todo gratis, porque de otro modo ni qué decir tiene que no se publicaria" (Corde, R. Gómez de la Serna: Automoribundia, 1948). 
ocurrencias muestran una progresión en el uso de estas formas, asi como la aparición de ni qué decir tiene (sic, con tilde) en el s. XX.

La búsqueda en CORDE de ni que decir, sin tilde, arroja más resultados. En los siglos iniciales aparece como construcción libre. Y solo hay un uso en el XIX de ni que decir tiene. Todos los restantes casos son del s. XX. Este operador es el que parece triunfar desplazando al inicio estos usos escalares de ni qué decir.

\begin{tabular}{|c|c|c|c|c|}
\hline NI QUE DECIR & $\begin{array}{c}\text { Construcción } \\
\text { libre }\end{array}$ & $\begin{array}{c}\text { Con comple- } \\
\text { mento }\end{array}$ & Operador & Ni que decir tiene \\
\hline $\mathrm{XVI}$ & 3 & & & \\
\hline $\mathrm{XVII}$ & 2 & & & \\
\hline $\mathrm{XVIII}$ & 1 & & & $1(1898)$ \\
\hline $\mathrm{XIX}$ & 1 & & & $18 / 48$ \\
\hline $\mathrm{XX}$ & $1 / 1$ & $4 / 9$ & $1 / 1$ & \\
\hline
\end{tabular}

Tabla 10: Ni que decir en CORDE

En inicial no se documenta hasta el s. XX, con mayor uso que el intercalado: 59 casos en inicial frente a 24 intercalado. Señalamos la distribución en el cuadro: el primer número corresponde a posición intercalada o final y el segundo en inicial. Hay que destacar que con estos usos iniciales aparece con valor reafirmativo y enlaza con la oración a través de que, como otros elementos de ese grupo. Por ello aparecen con complementos $(39,40)$ y algún uso como operador $(41)$ en intervención reactiva:

(39) - Es que... verá usted... Yo no quiero recibir auxilios de nadie sinó de Fernando. ¿Comprende? Ni que decir que le agradezco esas palabras tan amigas; pero... la verdad... si Fernando... (CORDE, S. González Anaya: La oración de la Tarde, 1929).

(40) Ni que decir que lo echaron a la calle como agua sucia. (idem).

(41) Nieves: ¿Para siempre, Serafin? Serafin: ¡Ni que decir! ¿Oyes? ¿No te embebece esa música? (Casi al oído.) Vamos al salón, que vean canela * (Entran en él.) (CORDE, C. Arniches: El amigo Melquiades o Por la boca muere el pez, 1914).

Ni que decir tiene aparece a finales del s. XIX (1898) y cobra gran vitalidad en el XX, como operador dominante:

(42) - Eso me disgusta - dijo Pío Cid-, pero puede que al fin no se vayan. Influya usted con ellos, aunque no sea más que por doña Paulita, que sabe usted que tiene un familión a su cargo.

-Eso ni que decir tiene- contestó Benito. (CORDE, A. Ganivet: Los trabajos del infatigable creador Pío Cid, 1898).

Los casos encontrados muestran que es frecuente la coordinación, manteniendo la construcción libre, al igual que ocurría en los otros corpus, y se mantiene, de manera más frecuente en los primeros siglos (desde el XVI). Los casos de operador ya surgen en el XX. Pero hay 
una diferencia entre ni qué hablary ni qué decir. En su origen aparece más ni qué decir o ni que decir que ni qué hablar. Hay solo 5 casos de la construcción libre con contenido léxico. No existe ni que hablar tiene, solo con decir (ni que decir tiene), y aparece desde temprano y de manera frecuente.

En cuanto a sus funciones macrosintácticas como operador, existe una diferencia clara entre dos contenidos:

-Reafirmativo: ni qué decir y ni que decir tiene / ni qué hablar. -Escalar: ni qué hablar (en enumeraciones).

Podemos decir, pues, que no son dos variantes equivalentes.

\section{Conclusión}

El análisis realizado sobre los corpus sincrónicos y diacrónico de la RAE nos lleva a afirmar que estamos ante una construcción que presenta variación en la forma verbal de base y que se encuentra en proceso de fijación. Podemos hablar de la coexistencia de tres patrones:

- La construcción libre [ni qué hablar / decir], integrada en la oración.

- La construcción semilibre [Ni qué hablar (decir) de + s.n. / que + oración]. En ella se desarrolla un contenido procedimental con contenido escalar y evidencial. Presenta lo que sigue como algo de lo que no hay dudas y que está situado en posición elevada de la escala.

- El operador discursivo ni qué hablar / ni qué decir, aún en proceso de fijación en el sistema. Generalmente diferencian sus usos. Ni qué hablar presenta fundamentalmente el contenido escalar y ni qué decir se especializa en el reafirmativo. Este, además, confluye con ni que decir tiene, operador argumentativo fijado.

Las formas con decir son más abundantes que con hablar, pero en la situación actual ambas están activas en el sistema.

Como pretendiamos mostrar con el trabajo, la génesis de nuevos operadores discursivos tiene una gran vitalidad, pero el proceso parece ir fijándose a través de etapas constructivas que actúan, de manera progresiva, tanto en lo formal, distribucional, como en el contenido procedimental que van desarrollando. El estadio de construcción semilibre es fundamental para poder explicar el proceso de "cooptación" (Heine, 2013) de estos operadores. 


\section{REFERENCIAS}

AIKHENVALD, A. Y. (2005): Evidentiality, Oxford: Oxford University Press.

ANSCOMBRE, J. C. \& DUCROT, O. (1983) : L'argumentation dans la langue, Liège: Pierre-Mardaga.

BERRENDONNER, A. (1990): "Pour une macro-syntaxe", Willems, D. (ed.), Données orales et théories linguistiques. Travaux de Linguistique, 21, Paris: Louvain Duculot, pp. 25-36.

BERRENDONNER, A. (2002): "Les deux syntaxes", Verbum, 24(12), pp. 23-36.

BLANCHE-BENVENISTE, C. (2003): "Le recouvrement de la syntaxe et de la macrosyntaxe", Scarano, A. (ed.): Macro-syntaxe et pragmatique. L'analyse linguistique de l'oral, Roma: Bulzoni Editore, pp. 53-75.

CASADO, M., LOUREDA, O. \& GONZÁLEZ, R. (2005): Estudios sobre lo metalingüístico (en español). Berne: Peter Lang.

COMPANY, C. (2004): “¿Gramaticalización o desgramaticalización? Reanálisis y subjetivización de verbos como marcadores discursivos en la historia del español", Revista de Filología Española, 84(1), pp. 29-66.

CORNILLIE, B. (2009): "Evidentiality and epistemic modality: On the close relationship between two different categories", Functions of Language, 16(1), pp. 44-62.

CORNILLIE, B. \& IZQUIERDO, D. (eds.) (2017): Gramática, semántica y pragmática de la evidencialidad, Pamplona: EUNSA.

CORPAS, G. (1996): Manual de fraseología española, Madrid: Gredos.

DELANCEY, S. (1997): "Mirativity: the gramatical marking of unexpected information", Linguistic Typology, 1, pp. 33-52.

DUCROT, O. (1980): Les échelles argumentatives, Paris: Minuit.

DUCROT, O. (1983): “Opérateurs argumentatives et visée argumentative", Cahiers de linguistique française, 5, pp. 7-36.

DUCROT, O. (1984): El decir y lo dicho, Buenos Aires: Hachette.

EVANS, N. (2007): "Insubordination and its uses", Nikolavea, I. (ed.): Finiteness, Oxford: Oxford University Press, pp. 366-431.

FAUCONNIER, G. (1975): "Pragmatic Scales and Logical Structure", Linguistic Inquiry, 6(3), pp. 353-375.

FERNÁNDEZ BERNÁRDEZ, C. (2002): Expresiones metalingüisticas con el verbo decir, A Coruña: Universidad da Coruña.

FERRARI, L. D. (2009): "Marcadores de modalidad epistémica y evidencial en el análisis de las conclusiones de artículos de investigación de disciplinas distintas", ALED, 9(2), pp. 5-23.

FRASER, B. (1996) "Pragmatic Markers", Pragmatics, 6(2), pp. 167-190.

FRASER, B. (1999): "What are Discourse Markers?", Journal of Pragmatics, 31, pp. 931-952.

FUENTES RODRÍGUEZ, C. (1991): "Adverbios de modalidad", Verba, 18, pp. 275-321.

FUENTES RODRÍGUEZ, C. (2003): "Operador/Conector, un Criterio para la Sintaxis Discursiva", RILCE, 19(1), pp. 61-85.

FUENTES RODRÍGUEZ, C. (2004): "Enunciación, modalidad y aserción, tres clásicos", Anuario de Estudios Filológicos, XXVII, pp. 121-145.

FUENTES RODRÍGUEZ, C. (2013): "La gramática discursiva: ni- 
veles, unidades y planos de análisis", Cuadernos AISPI, 2, pp. 15-36.

FUENTES RODRÍGUEZ, C. (2016): "Operadores discursivos: focalización, modalidad e intersubjetividad", López Serena, A., Narbona Jiménez, A. y Del Rey Quesada, S. (coords): El español a través del tiempo. Estudios ofrecidos a Rafael Cano Aguilar, Sevilla: Editorial Universidad de Sevilla, pp. 877-899.

FUENTES RODRÍGUEZ， C. (2017 [2000]): Lingüistica Pragmática y Análisis del discurso, Madrid: Arco Libros.

FUENTES RODRÍGUEZ, C. (2018): Parentéticos, Madrid: Arco Libros.

FUENTES RODRÍGUEZ, C. (2018 [2009]): Diccionario de conectores y operadores del español, $2^{\mathrm{a}}$ ed. Madrid: Arco Libros.

FUENTES RODRÍGUEZ, C. (2019): "Categorias discursivas y segmentación en macrosintaxis", Fuentes Rodríguez, C. \& Gutiérrez Ordóñez, S.: Avances en macrosintaxis, Madrid: Arco Libros, pp. 15-65.

FUENTES RODRÍGUEZ, C. (coord.) (2020): Operadores en proceso, Munich: Lincom.

FUENTES RODRÍGUEZ, C. (2021, e.p.): "La construcción y su rentabilidad en el ámbito de la macrosintaxis", Fuentes, C. et al. (eds): Construcciones y operadores discursivos del español, Sevilla: EUS.

FUENTES RODRÍGUEZ, C. \& ALCAIDE LARA, E. (1996): La expresión de la modalidad en el habla de Sevilla, Excmo. Ayuntamiento de Sevilla.

GOLDBERG, A. E. (1995): Constructions. A Construction Grammar Approach to Argument
Structure, Chicago / London: University of Chicago Press.

GOLDBERG, A. E. (2003): "Constructions: a new theoretical approach to language", TRENDS in Cognitive Sciences, 7(5), pp. 219-224. GOLDBERG, A. \& JACKENDOFF, R. (2004): “The English resultative as a family of constructions", Language, 80(3), 532568.

GRAS, P. \& SANSIÑENA, M. S. (2015): "An interactional account of discourse-connective que-constructions in Spanish", Text\&Talk, 35(4), pp. 505-529.

GREENBAUM, S. (1969): Studies in English Adverbial Usage, London: Longman.

HEINE, B. (2013): "On Discourse Markers: Grammaticalization, Pragmaticalization, or Something Else?", Linguistics, 5(6), pp. 1205-1247.

HOPPER, P. J. \& TRAUGOTT, E. C. (2003): Grammaticalization, Cambridge: Cambridge University Press.

LEHMANN, C. (1985): "Grammaticalization: Synchronic Variation and Diachronic Change", Lingua e Stile, 20(3), pp. 303-318.

LEHMANN, C. (2002): "New Reflections on Grammaticalization and Lexicalization", Wischer, I. y Diewald, G. M. (eds): New Reflections on Grammaticalization, Amsterdam: John Benjamins, pp. 1-18. doi:10.1075/ ts1.49.03leh.

MARTÍ SÁNCHEZ, M. (1999): "Problemas e implicaciones teóricas en el estudio de ni", Fernández González, J. et al. (eds.): Lingüistica para el siglo XXI, vol. II, Salamanca: Ediciones Universidad de Salamanca, pp. 1095-1100.

MARTÍ SÁNCHEZ, M. (2020): "Cons- 
trucciones fraseológicas y frasemas gramaticales con ni incoordinado", Romanica Olomucensia, 32(1), pp. 111-126, doi: $10.5507 /$ ro.2020.006.

MOECHSLER, J. \& RÉBOUL, A. (1994): Dictionnaire Encyclopédique de Pragmatique, Paris: Seuil.

NUYTS, J. (2001): Epistemic Modality. Language and Conceptualization, Amsterdam/ Philadelphia: John Benjamins Publishing Company.

PLUNGIAN, V. (2010): "Types of verbal evidentiality marking: an overview", Diewald, G. y Smirnova, E. (eds.): Linguistic Realization of Evidentiality in European Languages, Berlín/ Nueva York: De Gruyter Mouton, pp. 15-58.

PORTOLÉS, J. (2007): "Las escalas informativas convocadas por "ni" y "ni siquiera", Revista internacional de lingüistica iberoamericana, 10, pp. 199-220.

QUIRK, R. et al. (1972): A Comprehensive Grammar of the English Language, London: Longman.

RAMOS SAÑUDO, A. M. (2017): La fraseología del "decir" en francés: estudio de los marcadores de modalización, Tesis doctoral. Universidad de Sevilla.

REAL ACADEMIA ESPAÑOLA \& ASOCIACIÓN DE ACADEMIAS DE LA LENGUA ESPAÑOLA (2009): Nueva gramática de la lengua española. Morfología $y$ sintaxis, Madrid: Espasa.
RÉCANATI, F. (1979): La transparence et l'énonciation, Paris: Seuil.

TORDESILLAS, M. (1993): "Conectores y operadores: una diferencia de dinámica argumentativa", Revista de Filología Francesa, 3, pp. 233-44.

TRAUGOTT, E. C. (1995): "The Role of the Development of Discourse Markers in a Theory of Grammaticalization". Paper presented at ICHL XII, Manchester. Available online at: http:// www.stanford.edu/ traugott/papers/discourse. pdf (accessed 10 June 2014).

TRAUGOTT, E. C. (2010): "Grammaticalization", Jucker, A. H. y Taavitsainen, I. (eds.): Historical Pragmatics, Berlin: De Gruyter Mouton, pp. 97-126.

TRAUGOTT, E. \& TROUSDALE, G. (2013): Constructionalization and Constructional Changes, Oxford: Oxford University Press.

WILSON, D. (2016): "Reassessing the conceptual-procedural distinction", Lingua, 175-176, pp. 5-19. 